\title{
Quantum Statistics and Lasers
}

\author{
J. P. Gordon \\ Bell Telephone Laboratories, Inc., Murray Hill, N. J.
}

(Received February 26, 1964)

\begin{abstract}
We consider the quantum statistical features of a linear process of amplification or attenuation. The results are then used to estimate the spontaneous fluctuations of a class of laser oscillators. Finally, the question of a quantum mechanical information theory is discussed.
\end{abstract}

\section{Introduction}

In any discussion of the statistical properties of laser radiation, or indeed of any optical frequency radiation, if is of course important to keep in mind that the photon energy $h \nu$ is considerably larger than the classical thermal equipartition energy $k T$, for any temperature $T$ normally encountered in the laboratory. Hence the quantum nature of the radiation field is a necessary consideration. On the other hand, the radiation fields produced by laser oscillators might be expected to have much in common with the fields produced by common microwave oscillators, and it is useful to keep these parallels in mind also. In the present discussion, we will use the results of a quantum statistical calculation of a linear amplification process to illustrate such features.

In treating the radiation field in a finite volume of space, it is often convenient to analyze the field into a complete countable set of orthogonal modes [Heitler, 1953], each of which, neglecting its interaction with the material particles present, satisfies a harmonic oscillator type of equation of motion. For example, in a transmission line consisting of a periodic sequence of lenses, there is a countable set of transverse modes [Goubau and Schwering, 1961]; the spatial dependence of the field of each such mode along the transmission line in some finite length $L$ may then be Fourier analyzed into an orthogonal set of harmonic longitudinal modes.

Let us confine our attention now to the field of a single transverse mode with a given polarization. Let the quantity $v(t)$ (of dimension of voltage) be proportional to the electric field strength at some position along the line; the statistical properties of $v(t)$ are given by the characteristic function [Messiah, 1961]

$$
C[v(t)] \equiv\langle\exp \{i \xi v(t)\}\rangle
$$

where the angular brackets indicate a statistical average over a representative ensemble of identical systems, and where $i \equiv \sqrt{-1}$, and $\xi$ is a real parameter. The cumulant expansion [Kubo, 1962] of the characteristic function, in terms of the moments of the distribution of $v(t)$ over the ensemble, is

$$
\begin{gathered}
C[v(t)]=\exp \{i \xi\langle v(t)> \\
-\frac{\xi^{2}}{2 !}\left[\left\langle v^{2}(t)>-\langle v(t)\rangle^{2}\right]+\ldots\right\} .
\end{gathered}
$$

If we write $\sigma^{2}(t)=\left\langle v^{2}(t)\right\rangle-\langle v(t)\rangle^{2}$; i.e., $\sigma^{2}$ is the variance of the probability distribution of $v(t)$, then

$$
C[v(t)]=\exp \left\{i \xi<v(t)>-\frac{\xi^{2}}{2 !} \sigma^{2}(t)+\ldots\right\} .
$$

If $v(t)$ has a Gaussian distribution about its mean, then the cumulant expansion terminates after the second term.

Suppose that

$$
v(t)=v_{s}(t)+v_{n}(t),
$$

where $v_{s}(t)$ represents a signal, while $v_{n}(t)$ represents an additive stationary Gaussian noise. Then we can immediately identify

$$
\begin{aligned}
\langle v(t)\rangle & =v_{s}(t) \\
\sigma^{2} & =\left\langle v_{n}^{2}(t)\right\rangle
\end{aligned}
$$

That $\sigma^{2}$ is time independent follows from the assumed stationarity of the noise. Suppose also that the noise is white over some small band $\Delta \nu$ of interest near frequency $\nu$. Then the noise in this band is describable in terms of a temperature $T_{n}$ according to

$$
\frac{\sigma^{2}}{Z}=\frac{\left\langle v_{n}^{2}(t)\right\rangle}{Z}=\frac{1}{2} h \nu \Delta \nu \operatorname{coth}\left(\frac{h \nu}{2 k T_{n}}\right)
$$

where $Z$ is a proportionality constant with dimensions of resistance. Note that (5) is fully quantum mechanical; for $2 k T<<h \nu$ it reduces to the zero point power $\frac{1}{2} h \nu \Delta \nu$, while for $2 k T>>h \nu$ it reduces to the classical thermal power $k T \Delta \nu$.

Suppose now that the field in the line undergoes a linear process of amplification or attenuation through interaction with a large number of atoms maintained in a distribution corresponding respectively to a negative or positive Boltzmann 
temperature $T_{a}$. To avoid boundary problems (i.e., impedance mismatches, etc.) we can suppose that the atoms fill the line uniformly but that the interaction is turned on for some finite time $\tau$. A proper quantum mechanical calculation [Gordon, Louisell, and WaIker, 1963] has shown that if, in the absence of the interaction, the characteristic function would have been

$$
C_{0}[v(t)]=\exp \left\{i \xi v_{s}(t)-\frac{\xi^{2}}{2 !} \sigma^{2}\right\}
$$

with $\sigma^{2}$ given by (5), then, with the interaction, it becomes

$$
C[v(t)]=\exp \left\{i \xi \sqrt{G} v_{s}(t)-\frac{\xi^{2}}{2 !}\left[G \sigma^{2}+(1-G) \sigma_{1}^{2}\right]\right\}
$$

where $G$ is the power gain occasioned by the interaction, and $\sigma_{1}^{2}$ is an additional noise whose power is

$$
\frac{\sigma_{1}^{2}}{Z}=\frac{1}{2} h \nu \Delta \nu \operatorname{coth}\left(\frac{h \nu}{2 k T_{a}}\right)
$$

For simplicity we have here neglected the effects of dispersion. Expression (6) is valid both for amplification processes $\left(G>1, T_{a}<0\right)$ or attenuation processes $\left(G<1, T_{a}>0\right)$. We see that the signal is amplified in the classical way; i.e. "coherently"; the input noise, including the zero point noise, is amplified in the classical way, and there is an additional Gaussian noise, arising from the interaction, which makes the oritput signal to noise ratio always smaller than that of the input. ${ }^{1}$ For a high gain amplifier $\left(G>>1, T_{a}<0\right)$ the output noise power takes on the form

$$
G \frac{1}{2} h \nu \Delta \nu\left[\operatorname{coth}\left(\frac{h \nu}{2 k T_{n}}\right)+\operatorname{coth}\left(\frac{h \nu}{2 k\left|T_{a}\right|}\right)\right] .
$$

The smallest value this expression can take on is when

$$
T_{n}, T_{a} \rightarrow 0
$$

for which the output noise is

\section{$G h \nu \Delta \nu$}

This noise is physically the amplified spontaneous emission of the excited atoms; one can consider it as half arising from the amplification of the input zero point field and half arising internally within the atoms. It is useful to remember that the spontaneous emission noise has the statistical properties of an additive Gaussian noise. Consistent with this result for noise in the transmission line is a wire circuit picture where with each resistance $R$ is associated in series a random Gaussian noise voltage generator of magnitude

1 When one is considering measurements of voltage, or equivalently, of field When one is considering measurements on the properties of an additive Gaussian strength, then the zero point field takes on the properties of an additive Gaussian
noise field. However, it does not always assume this role. For example, it has noise field.

$$
\left\langle v_{n}^{2}\right\rangle=2 R h \nu \Delta \nu \operatorname{coth}\left(\frac{h \nu}{2 k T}\right) .
$$

This expression is again fully quantum mechanical, includes the zero point noise, and is valid for both positive and negative resistances (a negative resistance must have a negative temperature, so $\left\langle v_{n}^{2}\right\rangle$ is always positive).

Let us now consider a simple oscillator, consisting of a saturable negative resistance attached across a tuned circuit. If the negative resistance is initially larger in magnitude than the positive resistance of the tuned circuit, then oscillations build up in the circuit until the negative resistance decreases in magnitude to equal the positive resistance. This requirement determines the oscillation power level. If we assume now that the noise sources are not appreciably changed because of the necessary nonlinearity, then we can compute the fluctuations in the oscillator around its steady state following existing methods [Edson, 1960]. A principle result of such a calculation is that the oscillator phase undergoes a random walk type fluctuation, whose mean square shift is one radian in a "coherence" time given by

$$
\tau_{c}=\frac{1}{\Delta \omega} \frac{2 W_{0}}{h \nu\left[\operatorname{coth}\left(\frac{h \nu}{2 k T_{R}}\right)+\operatorname{coth}\left(\frac{h \nu}{2 k\left|T_{-R}\right|}\right)\right]}
$$

where $W_{0}$ is the energy stored in the circuit due to the oscillation, $\Delta \omega$ is the half width of the circuit $(\Delta \omega=\omega / 2 Q)$ in the absence of the negative resistance, and $T_{R}$ and $T_{-R}$ are the Boltzmann temperatures of the positive and negative resistances. In the limit of low temperature or high frequencies

$$
\tau_{c} \rightarrow \frac{1}{\Delta \omega} \frac{W_{0}}{h \nu}
$$

In most oscillators, lasers included, other effects; i.e., microphonic noise, temperature drifts, etc., cause phase fluctuations considerably greater than this, so (9) is probably only of academic interest.

At this point we would like to change the subject and speak briefly about information theory. In Shannon's mathematical theory of information [Shannon and Weaver, 1949], entropy plays a leading role. For the case of a signal accompanied by additive white Gaussian noise in a bandwith $B$ in a single mode transmission line, Shannon obtained his famous formula for information capacity (maximum attainable information rate)

$$
C=B \log _{2}\left(1+\frac{S}{k T B}\right) \text { bits/sec., }
$$

where $S$ is the average signal power and $k T B$ the noise power, both taken at the receiver input. Unfortunately no simple modification of this formula can make it correct in the realm of $h_{\nu}>k T$. Based 
on entropy considerations, however, the author [Gordon, 1962] has proposed a similar quantum formulation

$$
\begin{aligned}
& C<B \log _{2}\left(1+\frac{S}{N+h \nu B}\right) \\
&+\frac{S+N}{h \nu} \log _{2}\left(1+\frac{h \nu B}{S+N}\right) \\
& \quad-\frac{N}{h \nu} \log _{2}\left(1+\frac{h \nu B}{N}\right) .
\end{aligned}
$$

In this expression $N$ is the noise power in the mode neglecting the zero point field; i.e., in terms of an equivalent temperature

$$
\begin{aligned}
N & =\frac{1}{2} h \nu B\left[\operatorname{coth}\left(\frac{h \nu}{2 k T}\right)-1\right] \\
= & \frac{h \nu B}{\exp \left(\frac{h \nu}{k T}\right)-1} .
\end{aligned}
$$

Some discussion of (11) is in order. In the limit $N>>h_{\nu} B$ the second two terms drop out and the first reduces to Shannon's expression (10). In fact, the first term is a capacity when the first element of the receiver is a high gain maser amplifier. To show this we note that Shannon's formula should apply to the maser amplifier's output, where the best signal to noise ratio possible (with $\left|T_{a}\right| \rightarrow 0$ ) is just

$$
\frac{S}{N+h \nu B}
$$

which implies an information capacity of

$$
C_{\mathrm{amp}}=B \log \left(1+\frac{S}{N+h \nu B}\right) \text {. }
$$

However, in the limit of small powers

$$
h_{\nu} B>>S>>N
$$

the second term of (11) becomes dominant and can be much greater than $C_{\mathrm{amp}}$. Hence in this situation the best receiver does not involve an amplifier. Rather, one can obtain an information rate approaching the upper limit (11) by use of straight energy detection; i.e., photon counting. This is discussed in some detail by Gordon [1962].

The general problem of writing quantum mechanics into information theory is a relatively untouched area. Equation (11), while the author believes that it is correct, has not been proved, nor has any other expression been proposed. There are some fundamental things to be done here which have their roots in Shannon's work and in the quantum theory of measurements.

\section{References}

Edson, W. A. (Aug. 1960), Noise in oscillators, Proc. IRE 48, $1454-1466$.

Gordon, J. P. (Sept. 1962), Quantum effects in communications systems, Proc. IRE 50, No. 9, 1898-1908.

Gordon, J. P., W. H. Louisell, and L. R. Walker (April 1963)., Quantum statisties of masers and attenuators, Phys. Rev. 130, $806-812$.

Goubau, G., and F. Schwering (May 1961), On the guided propagation of electromagnetic wave beams, IRE Trans. Ant. Prop. AP-9, 248-255.

Heitler, W. (1953), The quantum theory or radiation, 3d ed. ch. 1 (Oxford University Press, Oxford, Eng.).

Kubo, R. (July 1962), Generalized cumulant expansion method, J. Phys. Soc. Japan 17, 1100-1120.

Messiah, A. (1961), Quantum Mechanies, 1, 449-450 (North Holland Pub. Co., Amsterdam).

Shannon, C. E., and W. Weaver (1949), The mathematical theory of communication (University of Illinois Press, Urbana, Ill.). 